\title{
Função renal, hepática e cardíaca de cães hígidos sob terapia prolongada com celecoxibe
}

\author{
Renal, hepatic and cardiac function in healthy dogs during long-term celecoxib therapy

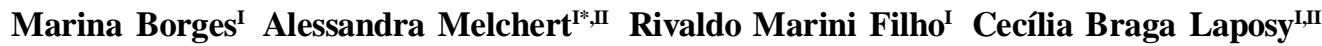 \\ Beatriz Cristina Conceição Porto Alegre ${ }^{\text {II }}$ Karine Ramires Silva ${ }^{\text {II }}$
}

\section{RESUMO}

O presente estudo teve como objetivo avaliar os efeitos da terapia prolongada com celecoxibe sobre a função renal, hepática e cardíaca em cães hígidos. Foram utilizados 12 cães fêmeas, divididos em 2 grupos: Gcelecoxibe: terapia com celecoxibe, na dose de $5 \mathrm{mg} \mathrm{kg}^{-1}$ por via oral, a cada 12

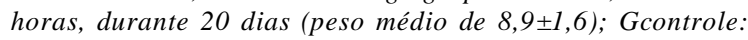
terapia com placebo, a cada 12 horas, por via oral, 20 dias

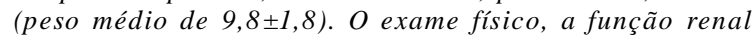
(urinálise; gamaglutamil transpeptidase -GGT e sódio urinários; ureia, creatinina, sódio e potássio séricos; e clearance endógeno de creatinina), tempo de coagulação (TC), biomarcadores cardíacos (creatinofosfoquinase - $C K$ e creatinofosfoquinase fração $M B-C K-M B)$ e função hepática (alanina aminotransferase -ALT, aspartato aminotransferase -AST e albumina) foram avaliados antes, aos 5, 10 e 20 dias (T0, T5, T10 e T20) do tratamento. No Gcelecoxibe, os valores de clearance de creatinina revelaram diminuição significativa no $\mathrm{T20}$, em relação ao $\mathrm{TO}$ e $\mathrm{T5}$, bem como redução em relação ao Gcontrole em T10 e T20. A urinálise, sódio, potássio, ureia e creatinina séricos, enzima GGT urinária e o TC não apresentaram variação entre os momentos ou grupos avaliados. Houve aumento significativo de CK-MB no T20 e ALT no T5, $T 10$ e T20 no Gcelecoxibe, entretanto, com valores dentro da normalidade para cães. Conclui-se que o celecoxibe revelouse seguro em relação ao perfil cardíaco e hepático em cães sadios, mesmo sob terapia prolongada. Sob vigência de terapia prolongada, deve ser usado cautelosamente em cães portadores de alterações renais.

Palavras-chave: celecoxibe, cão, função renal, função hepática, biomarcadores cardíacos.

\section{ABSTRACT}

The aim of this study was to evaluate the effects of long-term celecoxib therapy on renal, hepatic and cardiac profiles in healthy dogs. Twelve female were randomly assigned to 2 groups $(G)$ : Gcelecoxib: treated with celecoxib orally $\left(5 \mathrm{mg} \mathrm{kg}^{-1}\right)$, every 12 hours, for 20 days $(8.9 \pm 1.6$ body weight); Gcontrol: received placebo orally, every 12 hours, for 20 days $(9.8 \pm 1.8$ body weight). Physical examination, renal function (urinalysis, urinary sodium and gamma-glutamyl transpeptidase -GGT), serum urea, creatinine, potassium and sodium, and endogenous creatinine clearance), clotting time $(C T)$, cardiac biomarkers (creatine phosphokinase $-C K$ and $C K-M B$ ) and liver function (aspartate aminotransferase -AST, alanine aminotransferase $A L T$ and albumin) were evaluated before, at 5, 10 and 20 days (T0, T5, T10 and T20) of treatment. The creatinine clearance values showed significant decrease at $T 20$, in relation to $T 0$ and $T 5$ in the Gcelecoxib, and reduction in relation to Gcontrol at T10 and T20. The urinalysis, values of sodium, potassium, urea and creatinine serum and urinary GGT enzyme showed no difference through the study between moments or groups. There was a significant increase on CK values at T20 and on ALT values at T5, T10 and T2O in the Gcelecoxib, however with normal range values for dogs. Celecoxib revealed to be safe in relation to cardiac and hepatic profiles, even under prolonged therapy. However, it should be used judiciously during longterm therapy in dogs with renal dysunction.

Key words: celecoxib, dogs, renal function, hepatic function, cardiac biomarkers.

\section{INTRODUÇÃO}

Os anti-inflamatórios não-esteroidais (AINEs) são drogas amplamente utilizadas na clínica de pequenos animais. Atuam inibindo a síntese de mediadores químicos, basicamente atuando na inibição

IPrograma de Mestrado em Ciência Animal, Universidade do Oeste Paulista (UNOESTE), Rodovia Raposo Tavares, Km 572, 19067-175, Bairro Limoeiro, Presidente Prudente, SP, Brasil. E-mail: alessandravet@unoeste.br. *Autor para correspondência. "Curso de Medicina Veterinária, Faculdade de Ciências Agrárias, UNOESTE, Presidente Prudente, SP, Brasil. 
da enzima cicloxigenase (COX), que acarretará na diminuição de endoperóxidos cíclicos, tais como prostaglandinas (PG), prostaciclinas e tromboxanos (TX), importantes na mediação da dor e inflamação (JERICÓ \& ANDRADE, 2008). Várias são as ações terapêuticas dos AINEs, de caráter periférico, como as ações anti-inflamatórias, analgésicas, antitrombóticas e antiendotóxicas, ou sobre o sistema nervoso central (SNC), promovendo ação antipirética e também analgésica (KUMMER \& COELHO, 2002).

A enzima COX apresenta duas isoformas, intituladas COX-1 e COX-2 (KUMMER \& COELHO, 2002). A maioria dos AINEs inibe indiscriminadamente a COX-1 e a COX-2. A premissa inicial era de que a COX-1 seria uma enzima constitutiva envolvida em processos fisiológicos, como proteção da mucosa GI e vasodilatação renal, enquanto a COX-2 seria uma enzima induzida, com expressão aumentada em processos inflamatórios. Desse modo, foram desenvolvidos compostos seletivos para a inibição da COX-2 (coxibs), com o objetivo de proporcionar eficácia similar e maior segurança(VASCONCELOS et al., 2005).

Entretanto, estudos posteriores demonstraram que a COX-1 está presente juntamente com COX- 2 em sítios de inflamação na membrana sinovial, enquanto a COX-2 pode ser expressa constitutivamente (VASCONCELOS et al., 2005) em certas áreas do rim, cérebro e células endoteliais para as funções fisiológicas normais, o que demonstra atividade basal dessa isoforma nestes locais (PAPICH, 2008). Assim, a teoria clássica que distingue $\mathrm{COX}-1$ como uma enzima constitutiva e COX-2 como uma enzima induzida não é verdadeira (VASCONCELOS et al., 2005), colocando em discussão se os inibidores $\mathrm{COX}-2$ teriam realmente vantagens sobre os AINES convencionais (KUMMER \& COELHO, 2002).

Estão atribuídos a este grupo de fármacos efeitos adversos relatados no homem, tais como eventos cardiovasculares e tromboembólicos em usuários crônicos (CHAHADE et al., 2008). Ademais, a COX-2 atua no rim e a administração de drogas seletivas para COX-2 pode afetar adversamente o órgão em condições de desidratação, diminuição da perfusão ou doença renal. Por outro lado, com o uso de fármacos desta classe há favorável tolerabilidade gastrintestinal (PAPICH, 2008) e hepática (ENSMINGER et al., 2004).

$\mathrm{Na}$ medicina veterinária, em especial em pequenos animais, há carência de protocolos terapêuticos que padronizem o uso de inibidores COX-2 (ALENCAR et al., 2003). Sendo assim, o presente estudo teve como objetivo avaliar a função renal, biomarcadores cardíacos e enzimas hepáticas de cães hígidos submetidos à terapia prolongada (20 dias) com celecoxibe.

\section{MATERIAL E MÉTODOS}

Foram estudados 12 cães, sem raça definida, adultos, fêmeas, selecionados por normalidade dos exames físico e laboratorial, provenientes do canil da Instituição de origem. Os cães foram aleatoriamente divididos em 2 grupos ( $n=6)$ : Gcelecoxibe: peso médio inicial de $8,9 \pm 1,6 \mathrm{~kg}$, submetidos à administração de celecoxibe ${ }^{\text {a }}$, na dose de $5 \mathrm{mg} \mathrm{kg}^{-1}$, por via oral, a cada 12 horas, durante 20 dias; Gcontrole: peso médio inicial de $9,8 \pm 1,8 \mathrm{~kg}$, submetidos à administração de comprimido de placebo, a cada 12 horas, durante 20 dias. Durante o experimento, os cães foram mantidos em jaulas individuais de alvenaria, com água e ração comercial ad libitum.

O exame físico (coloração das mucosas, tempo de preenchimento capilar, temperatura retal $(\mathrm{T})$, frequências cardíaca (FC) e respiratória (FR), estado de hidratação e teste de sensibilidade renal, avaliado pelos mesmos avaliadores em sistema duplo cego, por meio de palpação, determinado por escore, sendo $1=$ negativo e 2 = positivo para dor renal), peso corporal, tempo de coagulação (TC), a função renal (urinálise; GGT e sódio urinários; ureia, creatinina, sódio e potássio séricos; e clearance endógeno de creatinina), os biomarcadores cardíacos (enzimas creatinofosfoquinase -CK e creatinofosfoquinase fração MB -CK-MB), e perfil hepático (enzimas aspartato amino-transferase -AST, alanina amino-transferase -ALT, fosfatase alcalina -FAe albumina sérica) foram avaliados antes, aos cinco, $10 \mathrm{e}$ 20 dias (T0, T5, T10 e T20) de tratamento. Diariamente, foi avaliado o apetite e fezes. $\mathrm{O}$ apetite foi avaliado pela ingestão de ração diária de ração pesada no cocho individual, considerando-se a indicação do fabricante de 15-20g de ração $\mathrm{kg}^{-1} \mathrm{dia}^{-1}$, classificado em escores comonormal (1), aumentado (2), diminuído (3) ou anorexia (4). As fezes foram avaliadas macroscopicamente em normais (1), pastosas (2), diarreia (3) ou com sangue (4).

Para as determinações bioquímicas sanguíneas, foi coletado sangue por venopunção jugular. A ureia foi determinada pelo método urease ponto final ${ }^{\mathrm{b}}$ e a creatinina pelo método do picrato alcalino ${ }^{\mathrm{b}}$. O sódio e potássio séricos e sódio urinário foram mensurados em analisador de eletrólitos ${ }^{\mathrm{c}}$ e determinados pelo método de íon seletivo (ISE). Os exames de FA, AST, ALT, CK e CK-MB foram realizados através do método cinético $\mathrm{UV}^{\mathrm{d}}$, dosados em analisador semi-automático ${ }^{\mathrm{e}}$. A albumina sérica foi determinada pelo método de verde de bromocresol ${ }^{\mathrm{d}}$. O TC foi determinado em segundos pelo método de Lee $\&$ White.

Amostras de urina foram colhidas por sondagem uretral (OSBORNE \& FINCO, 1995) para urinálise e determinação da enzima GGT urinária pelo 
método cinético $U^{\mathrm{f}}$. $\mathrm{O}$ clearance endógeno de creatinina foi realizado em dois períodos subsequentes de 20 minutos, realizado de acordo com OSBORNE \& FINCO, (1995). Após a cateterização, foi realizado completo esvaziamento e lavagem da bexiga urinária, iniciando-se então o tempo do teste. Após 20 minutos, foi coletada toda a urina produzida, com auxílio de lavagem da bexiga com solução fisiológica e monitorização por ultrassonografia para certificação do seu esvaziamento. Na metade do tempo do teste (10 minutos), foi coletado sangue para determinação da creatinina sérica. $\mathrm{O}$ clearance foi realizado por duas vezes consecutivas para maior confiabilidade do teste.

O clearance foi calculado multiplicando-se a concentração de creatinina urinária pelo volume urinário produzido em mililitros $(\mathrm{mL})$ por minuto $(\mathrm{min})$, utilizando-se a fórmula: TFG $\left(\mathrm{mL} \mathrm{min}^{-1}\right)=($ Urina cr $[\mathrm{mg}$ $\left.\mathrm{dL}^{-1}\right] \mathrm{X}$ Volume de urina $\left[\mathrm{mL} \mathrm{min}^{-1}\right] /$ Soro $\mathrm{cr}\left(\mathrm{mg} \mathrm{dL}^{-1}\right) . \mathrm{O}$ resultado foi dividido pelo peso do animal, obtendo-se a estimativa da TFG em mL min ${ }^{-1} \mathrm{~kg}^{-1}$ (GRAUER, 2001). Como foram realizados dois períodos de avaliação do clearance em cada momento, foi utilizada a média dos dois clearances para realização da análise estatística.

Para análise estatística, foi utilizado o método multivariado de perfil para amostras dependentes, para avaliação dos diferentes momentos estudados. Para as variáveis teste de sensibilidade renal, avaliação das mucosas, cor da urina, sangue oculto na urina, apetite e fezes, foi utilizado o modelo de análise estatística não paramétrica, com aplicação do teste de Friedman, para comparação dos momentos de avaliação. Para avaliação entre os dois grupos estudados, foi usado o teste de $\mathrm{T}$ não pareado. Foi considerado nível de 5\% de significância.

\section{RESULTADOS}

Não foram observadas alterações nos parâmetros avaliados no exame físico nos cães dos Grupos celecoxibe ou controle neste estudo, em todos os momentos avaliados. Nenhum dos animais apresentou evidências de dor ao teste de sensibilidade renal, sendo todos negativos à avaliação da dor por meio de palpação da região dos rins em ambos os grupos, em T0, T5, T10 e T20. Todos os animais apresentaram estado de hidratação adequado e mucosas normocoradas nos diferentes momentos e grupos avaliados. O peso corporal manteve-se constante durante todo o período de tratamento em ambos os grupos. Os pesos médios, em kg, dos cães do Gcelecoxibe foram $8,9 \pm 1,8 ; 8,8 \pm 1,3 ; 9,0 \pm 2,7$ e $9,1 \pm 2,6$ e do Gcontrole foram $9,8 \pm 1,8 ; 9,6 \pm 1,7 ; 9,7 \pm 1,4$ e $9,6 \pm 1,6$, para T0, T5, T10 e T20, respectivamente.
A FC, FR e T apresentaram-se dentro da normalidade para cães (FEITOSA, 2008), não sendo observadas diferenças significantes em nenhum dos parâmetros entre os momentos ou grupos estudados. Não foi constatada presença de vômitos, as fezes e o apetite apresentaram-se normais em todos os momentos, em ambos os grupos.

$\mathrm{Na}$ avaliação da função renal, a urinálise, creatinina e a enzima GGT urinárias não revelaram variação entre os momentos ou grupos. A excreção urinária de sódio não foi alterada e manteve-se dentro da normalidade, mesmo após os 20 dias de tratamento com celecoxibe, obtendo-se, neste grupo, valores de $90,2 \pm 28,9 ; 117,8 \pm 53,2 ; 93,2 \pm 87,5$ e 127,3 $\pm 24,9$, para T0, T5, T10 e T20, respectivamente, e valores de 96,5 $\pm 13,4$; $108,6 \pm 32,4 ; 106,3 \pm 42,9$ e $113,3 \pm 34,6$ no Gcontrole. Valores de $96 \pm 38 \mathrm{mmol} \mathrm{L}^{-1}$ foram considerados dentro da normalidade para cães (HINCHCLIFF et al., 1997).

Os valores de sódio, potássio, ureia e creatinina séricos não apresentaram diferença significativa entre os momentos ou grupos avaliados e mantiveram-se dentro da normalidade para cães (TRHALL, 2007; KANEKO et al., 2008) (tabela 1). Os valores de clearance endógeno de creatinina revelaram diminuição significativa no T20, em relação ao T0 e ao T5 no Gcelecoxibe, bem como redução em relação ao Gcontrole em T10 e T20 (tabela 1), com valores abaixo da normalidade para cães no T20 (OSBORNE \& FINCO, 1995). No Gcontrole, não foram observadas alterações do clearance entre os momentos estudados.

$\mathrm{Na}$ avaliação dos biomarcadores cardíacos, houve aumento de CK-MB no T20, em relação ao T0 no Gcelecoxibe, enquanto CK não apresentou diferença significativa entre os momentos ou grupos avaliados. Os valores de CK-MB mantiveram-se, entretanto, dentro da normalidade para cães (KANEKO et al., 2008) no Gcelecoxibe. No Gcontrole, não foram observadas alterações de CK-MB ou CK entre os diferentes momentos estudados (tabela 2). Na avaliação do TC, não foram observadas diferenças significantes no TC entre os momentos avaliados dentro de cada um dos grupos, bem como entre os dois grupos estudados (tabela 1).

Na função hepática, a enzima ALT revelou aumento no T5, T10 e T20 em relação ao T0 no Gcelecoxibe, bem como aumento significativo em relação ao Gcontrole no T5 e T20, mantendo-se dentro da normalidade (KANEKO et al., 2008), entretanto, em todos os momentos. As enzimas AST e FA, bem como a albumina sérica, não revelaram diferenças significantes quando confrontados os momentos ou grupos avaliados. Os valores médios e desvios padrão dos biomarcadores cardíacos e hepáticos dos grupos estudados em T0, T5, T10 e T20 estão representados na tabela 2 . 
Tabela 1- Médias e desvios-padrão dos parâmetros clearance endógeno de creatinina $\left(\mathrm{mL} \mathrm{min}^{-1} \mathrm{~kg}^{-1}\right), \mathrm{ureia}\left(\mathrm{mg} \mathrm{dL}^{-1}\right)$, creatinina (mg dL $\left.\mathrm{m}^{-1}\right)$, sódio $\left(\mathrm{mEq} \mathrm{L} \mathrm{L}^{-1}\right)$, potássio $\left(\mathrm{mEq} \mathrm{L}^{-1}\right)$ séricos e tempo de coagulação - $\mathrm{TC}$ (segundos) dos animais dos grupos Gcelecoxibe e Gcontrole, antes, aos 5, 10 e 20 dias de terapia.

\begin{tabular}{|c|c|c|c|c|c|}
\hline Parâmetros & Grupos & Antes & 5 dias & 10 dias & 20 dias \\
\hline \multirow[t]{2}{*}{ Clearance } & GCelecoxibe & $3,2 \pm 1,0$ & $3,4 \pm 0,6$ & $2,6 \pm 0,7^{*}$ & $2,0 \pm 0,8 *$ \\
\hline & GControle & $3,5 \pm 1,2$ & $3,7 \pm 0,6$ & $3,6 \pm 0,8$ & $3,7 \pm 0,5$ \\
\hline \multirow[t]{2}{*}{ Ureia } & GCelecoxibe & $39,9 \pm 11,4$ & $39,3 \pm 10,8$ & $40,6 \pm 9,3$ & $35,9 \pm 6,1$ \\
\hline & GControle & $38,9 \pm 12,3$ & $48,9 \pm 8,3$ & $45,8 \pm 11,5$ & $41,9 \pm 10,5$ \\
\hline \multirow[t]{2}{*}{ Creatinina } & GCelecoxibe & $1,0 \pm 0,1$ & $0,9 \pm 0,1$ & $0,9 \pm 0,1$ & $0,9 \pm 0,1$ \\
\hline & GControle & $0,9 \pm 0,1$ & $1,0 \pm 0,1$ & $0,9 \pm 0,1$ & $1,0 \pm 0,1$ \\
\hline \multirow[t]{2}{*}{ Sódio } & GCelecoxibe & $145,0 \pm 2,8$ & $145,3 \pm 2,0$ & $145,3 \pm 1,9$ & $145,7 \pm 1,4$ \\
\hline & GControle & $145,7 \pm 1,0$ & $144,8 \pm 0,8$ & $145,7 \pm 1,2$ & $145,5 \pm 1,6$ \\
\hline \multirow[t]{2}{*}{ Potássio } & GCelecoxibe & $4,4 \pm 0,2$ & $4,4 \pm 0,2$ & $4,5 \pm 0,3$ & $4,6 \pm 0,1$ \\
\hline & GControle & $4,4 \pm 0,2$ & $4,3 \pm 0,3$ & $4,4 \pm 0,3$ & $4,4 \pm 0,3$ \\
\hline \multirow[t]{2}{*}{$\mathrm{TC}$} & GCelecoxibe & $619,7 \pm 283,2$ & $614,7 \pm 198,0$ & $487,8 \pm 242,4$ & $641,0 \pm 97,1$ \\
\hline & GControle & $762,7 \pm 120,5$ & $698,5 \pm 70,2$ & $617,8 \pm 125,7$ & $639,3 \pm 168,4$ \\
\hline
\end{tabular}

* $(\mathrm{P}<0,05) \mathrm{X}$ Antes e 5 dias e $(\mathrm{P}<0,03) \mathrm{X}$ Gcontrole 20 dias.

* $(\mathrm{P}<0,03) \mathrm{X}$ Gcontrole 10 dias.

Parâmetros normais: Ureia 21-60 (mg dL $\left.{ }^{-1}\right)$; Creatinina 0,5-1,6 (mg dL $\left.{ }^{-1}\right)$ (KANEKO et al., 2008);

Sódio 145-158 (mEq L $\left.{ }^{-1}\right)$; Potássio 4,1-5,5 (mEq L $\left.{ }^{-1}\right)$; TC 300-900 (seg) (TRHALL, 2007);

Clearance endógeno de creatinina 2,66 $\pm 0,14\left(\mathrm{~mL} \mathrm{~min}^{-1} \mathrm{~kg}^{-1}\right)($ OSBORNE \& FINCO, 1995).

\section{DISCUSSÃO}

O uso do celecoxibe não promoveu alterações nos parâmetros do exame físico ou associadas ao trato GI. Mesmo após vinte dias de terapia não foram observadas alterações no apetite, vômitos, diarreia ou presença de sangue nas fezes, indicando a favorável tolerabilidade GI desse fármaco. A despeito de sua eficácia, o maior fator limitante do uso de AINEs é sua toxicidade GI, variando desde uma leve dispepsia a complicações graves, tais como úlceras pépticas, sangramentos, perfurações e obstruções (LAINE, 2001).

Entretanto, essa toxicidade GI parece estar relacionada com a inibição da função citoprotetora da COX-1. Assim, inibidores seletivos da COX-2 foram desenvolvidos com o objetivo de inibir a produção de PGs dependentes de COX-2, poupando COX-1 e sua função gastroprotetora (VASCONCELOS et al., 2005), fatos corroborados por este estudo. Entretanto, devese considerar que avaliação mais acurada do trato GI, como endoscópica, não foi realizada, o que não descarta a possibilidade da ocorrência de lesões GI ocultas.
Apesar dos inibidores seletivos da COX-2 apresentarem potente atividade anti-inflamatória in vivo e promoverem efeitos colaterais GI mínimos, tem sido demonstrado que podem produzir efeitos colaterais sobre os rins. Eventos adversos renais e renovasculares têm sido associados ao uso de AINES tradicionais (não seletivos) e aos inibidores da COX-2 (PAPICH, 2008). Os efeitos sobre o fluxo sanguíneo renal podem promover insuficiência renal reversível ou mesmo irreversível, naqueles pacientes com nefropatia associada a analgésicos, usados continuamente em doses altas (BRUNTON, 2010).

Os inibidores seletivos da COX-2 podem precipitar lesão renal em alguns pacientes. A frequência relativa dessa ocorrência comparada com os AINES não-seletivos ainda é desconhecida (BRADEN et al., 2004). Neste estudo, não foram observadas alterações na urinálise, creatinina ou GGT urinária. Entretanto, foi constatada redução do clearance de creatinina após 20 dias de terapia.

O rim depende da COX-1 e COX-2 para a síntese de PG, autorregulação de água e funções tubulares. A COX-2 é encontrada em várias seções do rim e a administração de AINEs COX-2 pode afetar 
Tabela 2 - Médias e desvios-padrão dos parâmetros albumina sérica (g/dL), enzimas ALT (U L $\left.L^{-1}\right), F$ ( $\left.\mathrm{U} \mathrm{L}^{-1}\right), \mathrm{CK}^{-M B}\left(\mathrm{U} \mathrm{L}^{-1}\right)$ e CK (U L $\left.{ }^{-1}\right)$ dos animais dos grupos Gcelecoxibe e Gcontrole, antes, aos 5, 10 e 20 dias de terapia.

\begin{tabular}{|c|c|c|c|c|c|}
\hline Parâmetros & Grupos & Antes & 5 dias & 10 dias & 20 dias \\
\hline \multirow[t]{2}{*}{ Albumina } & GCelecoxibe & $2,7 \pm 0,3$ & $2,9 \pm 0,5$ & $3,2 \pm 1,1$ & $2,8 \pm 0,6$ \\
\hline & GControle & $2,9 \pm 0,2$ & $2,8 \pm 0,2$ & $2,9 \pm 0,6$ & $3,0 \pm 0,5$ \\
\hline \multirow[t]{2}{*}{ ALT } & GCelecoxibe & $28,6 \pm 13,3$ & $44,0 \pm 22,3^{*}$ & $41,5 \pm 23,0^{*}$ & $41,3 \pm 23,3 * *$ \\
\hline & GControle & $31,5 \pm 8,5$ & $30,8 \pm 9,6$ & $35,5 \pm 8,5$ & $29,3 \pm 8,4$ \\
\hline \multirow[t]{2}{*}{ AST } & GCelecoxibe & $28,6 \pm 13,4$ & $44,0 \pm 22,0$ & $41,5 \pm 23,1$ & $41,3 \pm 23,0$ \\
\hline & GControle & $25,2 \pm 8,1$ & $34,8 \pm 7,2$ & $34,3 \pm 12,5$ & $30,0 \pm 17,9$ \\
\hline \multirow[t]{2}{*}{ FA } & GCelecoxibe & $37,0 \pm 19,9$ & $30,3 \pm 9,0$ & $29,8 \pm 11,3$ & $30,0 \pm 17,4$ \\
\hline & GControle & $34,4 \pm 17,9$ & $33,0 \pm 15,0$ & $27,8 \pm 12,2$ & $28,3 \pm 9,7$ \\
\hline \multirow[t]{2}{*}{ CK-MB } & GCelecoxibe & $14,0 \pm 4,4$ & $17,4 \pm 6,9$ & $18,2 \pm 6,1$ & $18,9 \pm 6,2 *$ \\
\hline & GControle & $16,7 \pm 2,4$ & $18,5 \pm 6,0$ & $15,7 \pm 4,1$ & $17,3 \pm 1,5$ \\
\hline \multirow[t]{2}{*}{$\mathrm{CK}$} & GCelecoxibe & $89,0 \pm 21,5$ & $101,3 \pm 46,6$ & $130,3 \pm 67,0$ & $99,3 \pm 39,0$ \\
\hline & GControle & $105,7 \pm 14,2$ & $119,3 \pm 36,9$ & $122,7 \pm 40,5$ & $99,0 \pm 10,8$ \\
\hline
\end{tabular}

* $(\mathrm{P}<0,05) \mathrm{X}$ Antes; $(\mathrm{P}<0,05) \mathrm{X}$ Gcontole 5 dias; $\bullet(\mathrm{P}<0,05) \mathrm{X}$ Gcontole 20 dias.

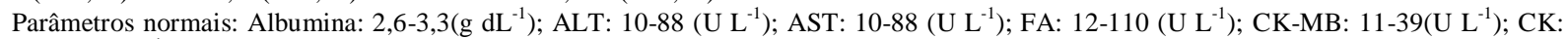
47-329 (U L $\left.{ }^{-1}\right)$ (KANEKO et al., 2008).

adversamente o órgão em condições de perfusão reduzida, tais como desidratação, anestesia, choque ou doença renal (PAPICH, 2008). Entretanto, mesmo na condição de higidez dos cães deste estudo, observou-se alteração da função renal sob terapia prolongada com celecoxibe, com redução dos valores de clearance em T20, constatando-se redução significativa da TFG, o que demonstra que o uso prolongado do fármaco a reduz e prejudica a função renal. O celecoxibe inibe a PG endotelial e a vasodilatação da arteríola aferente, causando a redução da TFG, pela inibição da ação da COX-2. Essa inibição pode causar síndrome nefrótica, levando à nefrite $\mathrm{e}$ consequente proteinúria (AUDIA, et al., 2008). Devese ter cautela ao fazer uso de terapias prolongadas com celecoxibe naqueles pacientes que apresentem fatores de risco para doença renal.

A toxicidade renal associada com AINEs é caracterizada pela diminuição da perfusão renal, retenção de sódio, fluido e função tubular diminuída (PAPICH, 2008). Entretanto, neste estudo não foi observada redução da excreção de sódio, nem tão pouco alterações da urinálise que revelassem alterações tubulares. Experimento em camundongos, que atribuiu a geração de PGs renais vasodilatadoras à COX-2, levantou a possibilidade de que a incidência de complicações hipertensivas induzidas pelos AINEs pode correlacionar-se com o grau de inibição da COX-2 no rim e com a seletividade dessa inibição (QI et al., 2006).

Em pacientes com doença glomerular, a inibição da síntese de PGs por AINES pode levar à isquemia renal reversível, declínio da pressão hidrostática glomerular e insuficiência renal aguda (IRA) (ROSE, 2007), uma vez que, nesses pacientes, o aumento na produção de $\mathrm{PGs}$ parece manter a taxa de filtração glomerular na presença de importante redução na permeabilidade do capilar glomerular (HUERTA et al., 2005). Desse modo, deve-se ter máxima cautela ao fazer uso do celecoxibe em pacientes com doenças renais pré-existentes.

Os inibidores seletivos da COX-2 vieram ao mercado com o objetivo de reduzir o risco de lesões gastrointestinais, entretanto, ocorrência elevada de eventos cardiovasculares e tromboembólicos foi relatada em pacientes humanos usuários crônicos desses fármacos (CHAHADE et al., 2008). Neste estudo, a principal alteração das enzimas cardíacas foi o aumento da CK-MB no T20, achado que não representou importância clínica, uma vez que os valores de CK-MB permaneceram dentro da normalidade para cães.

Um fato importante a considerar ao instituir terapia com AINEs é a ocorrência de alterações do perfil de coagulação, especialmente sob vigência de fármacos COX-2 seletivos. A homeostase cardiovascular é 
controlada pelo equilíbrio entre COX-1, dependente da produção de tromboxano (TX) A2 pelas plaquetas, que atua como potente ativador de plaquetas e vasoconstritor, promovendo agregação plaquetária, e COX-2, mediada pela produção de PG I2 pelas células endoteliais, que inibe a agregação plaquetária e formação de trombos. Os AINEs COX-2 seletivos reduzem a formação de PGI2 pelas células endoteliais, consequentemente rompendo o equilíbrio entre TXA2 e PGI2 e potencialmente favorecendo eventos prótrombóticos cardiovasculares (FRESNO et al., 2008).

Essas alterações da fisiologia da cascata do ácido aracdônico teoricamente explicam o aumento na ocorrência de eventos cardiovasculares associados ao uso de coxibes no homem (MONTEIRO et al., 2008). Entretanto, um estudo em cães revelou que o uso prolongado do firocoxibe por 28 dias não foi capaz de afetar o tempo de sangramento ou de produzir alterações da agregação plaquetária (STEAGALL et al., 2007). Deve-se atentar, entretanto, que, apesar de não haver diferenças significativas na análise do TC nos cães tratados com Celecoxibe, neste estudo, e de os valores encontrados em todas as avaliações estarem dentro dos valores de referência para cães, em T10, os valores revelaram-se menores que os valores registrados em T0, T5 e T20 e também que os do Gcontrole, devendo-se ter atenção quando do uso do fármaco em pacientes com tendência ao aumento da coagulabilidade sanguínea.

$\mathrm{Na}$ avaliação da função hepática, a enzima ALT revelou aumento significativo em todos os momentos avaliados (T5, T10 e T20), em relação ao momento controle (T0). A ALT e a AST podem ser considerados os exames de bioquímica sanguínea mais informativos para avaliação da toxicidade aguda por AINEs e sua elevação em conjunto pode indicar lesão hepática significativa (FOX \& CAMPBELL, 2000), o que não foi observado neste estudo, uma vez que os valores obtidos mantiveram-se dentro da normalidade para cães.

Os AINEs podem causar lesão hepática, sendo esta provocada mais por mecanismo idiossincrático que por efeito tóxico direto, o que não foi observado nos cães deste estudo. No homem, o aspecto clínico dessa lesão varia desde elevações assintomáticas das aminotransferases até quadros de hepatite aguda grave com icterícia hepatocelular, evoluindo para óbito (BARBOSA, 2008). Em contrapartida, ENSMINGER et al. (2004) referem que o uso de celecoxibe em cães submetidos a contraste tóxico para as vias biliares foi capaz de reduzir as injúrias induzidas pelo contraste, tais como necrose e esclerose.

\section{CONCLUSÃO}

Conclui-se que o celecoxibe revela-se seguro em relação ao perfil cardíaco e hepático em cães, mesmo sob terapia prolongada. Sob vigência de terapia prolongada (20 dias), deve ser usado cautelosamente em portadores de alterações renais, uma vez que foi capaz de reduzir o clearance endógeno de creatinina.

\section{APROVAÇÃO ÉTICA}

A pesquisa foi aprovada pelo Comitê de Ética em Pesquisa da instituição de origem, protocolada sob o no ${ }^{\circ}$. 056/OL.

\section{FONTES DE AQUISIÇÃO}

a - Celebra: celecoxibe, Laboratório Pfizer Pharmaceuceuticals LLC. Caguas, Porto Rico.

b - Bioclin: Quibasa Química Básica Ltda. Belo Horizonte, Brasil. c - AVL 9180 electrolyte analyzer: Roche Diagnostics. New York, USA.

d - Kit Labtest: Lagoa Santa, Brasil.

e - Aparelho Quick Lab II Drake: Gold Analisa Diagnóstica Ltda. Belo Horizonte, Brasil.

f - Gama GT Liquiform: Labtest: Lagoa Santa, Brasil.

\section{REFERÊNCIAS}

ALENCAR, M.M.A. et al. Margem de segurança do meloxicam em cães: efeitos deletérios nas células sangüíneas e trato gastrintestinal. Ciência Rural, Santa Maria v.33, n.3, p.525532, 2003. Disponível em: <http://www.scielo.br/pdf/cr/v33n3/ a21v33n3.pdf>. Acesso em: 18 ago. 2010. doi: 10.1590/ S0103-84782003000300021. ISSN 0103-8478.

AUDIA, P. et al. Metformina induzida acidose láctica e pancreatite aguda precipitada pelo celecoxib, diurético, e candesartan associado a disfunção renal aguda. Clinical Toxicology, New York, v.46, n.2, p.164-166, 2008.

BARBOSA, CM. Avaliações hematológicas e bioquímicas do uso de diclofenaco de sódio, meloxicam e firocoxibe em ratos wistar. 2008. 124f. Dissertação (Mestrado em Clínica Veterinária) - Faculdade de Medicina Veterinária e Zootecnia, Universidade Estadual Paulista, SP.

BRADEN, G.L. et al. Acute renal failure and hyperkalaemia associated with cyclooxygenase- 2 inhibitors. Nephrology Dialisys Transplantation, Oxford, v.19, n.5, p.1149-1153, 2004. Disponível em: <http://ndt.oxfordjournals.org/content/19/5/ 1149.full>. Acesso em: 20 nov. 2008. doi: 10.1093/ndt/gfg622.

BRUNTON, L. Goodman e Gilman: bases farmacológicas da terapêutica. 11.ed. Porto Alegre: AMGH, 2010. 1844p.

CHAHADE, W.H. et al. Antiinflamatórios não hormonais. Einstein, São Paulo, v.6, Supl.1, p.166-174, 2008.

ENSMINGER, W.J. et al. Effects of dexamethasone or celecoxib on biliary toxicity after hepatic arterial infusion of 5-fluorodeoxyuridine in a canine model. Cancer Research, Baltimore, v.64, n.1, p.311-315, 2004. Disponível em: <http:// 
cancerres.aacrjournals.org/content/64/1/311.full.pdf+html> . Acesso em: 14 set. 2009. doi:10.1158/0008-5472.CAN-03-2588.

FEITOSA, F.L.F. Exame físico geral ou de rotina. In: Semiologia veterinária - a arte do diagnóstico. 2.ed. São Paulo: Roca, 2008. Cap.4, p.65-86.

FOX, S.M.; CAMPBELL, S. Atualização: dois anos (19971998) de experiência clínica com rimadyl ${ }^{\circledR}$. Pfizer Saúde Animal-Boletim Técnico, São Paulo, v.1, p.4-6, 2000.

FRESNO, M. et al. Prostanoids actions in cardiovascular physiopathology. Anales de la Real Academia Nacional de Farmacia, Madrid, v.74, n.4, p.1-23, 2008.

GRAUER, G.F. Testes diagnósticos para o sistema urinário. In: NELSON, R.W.; COUTO, C.G. Medicina interna de pequenos animais. 2.ed. Rio de Janeiro: Guanabara Koogan, 2001. p.466-479.

HINCHCLIFF, K.W. et al. Exercise-associated hyponatremia in Alaskan sled dogs: urinary and hormonal responses. Journal of Applied Physiology, Bethesda, v.83, p.824-829, 1997.

HUERTA, C. et al. Nonsteroidal anti-inflammatory drugs and risk of ARF in the general population. American Journal of Kidney Diseases, Amsterdan, v.45, n.3, p.531-539, 2005.

JERICÓ, M.M.; ANDRADE, S.F. Antiinflamatórios. In: ANDRADE, S.F. Manual de Terapêutica Veterinária. 3.ed. São Paulo: Roca, 2008. Cap.7, p.115-140.

KANEKO J.J. et al. Clinical biochemistry of domestic animals. 6.ed. New York: Academic, 2008. 896p.

KUMMER, C.L.; COELHO, T.C. Cyclooxygenase -2 inhibitors nonsteroid anti-inflammatory drugs: current issues. Revista Brasileira de Anestesiologia, Rio de Janeiro, v.52, n.4, p.498-512, 2002 .
LAINE, L. Approaches to NSAID use in the high-risk patient. Gastroenterology, Amsterdan, v.120, p.594-606, 2001. Disponível em: <http://sadieo.ucsf.edu/course/old/pre-2005/ Lainegast.pdf $>$. Acesso em: 15 dez. 2009. doi:10.1053/ gast. 2001.21907 .

MONTEIRO, E.C.A. et al. Os antiinflamatórios não esteroidais (AINEs). Temas de Reumatologia Clínica, São Paulo, v.9, n.2, p.53-63, 2008.

OSBORNE, C.A.; FINCO, D.R. Canine and feline nephrology and urology. Baltmore: Lea \& Febiger, 1995. 960p.

PAPICH, M.G. An update on nonsteroidal anti-inflammatory drugs in small animals. Veterinary Clinics of North America - Small Animal Practice, Philadelphia, v.38, p.1243-1266, 2008 .

QI, Z. et al. Differentiation of Cyclooxygenase 1- and 2 - Derived Prostanoids in Mouse kidney and aorta. Hypernetsion, Dallas, v.48, p.323-328, 2006. Disponível em: <http://hyper.ahajournals.org/ content/48/2/323>. Acesso em: 06 ago. 2010. doi: 10.1161/ 01.HYP.0000231934.67549.b7.

ROSE, B.D. NSAIDs: acute renal failure and nephrotic syndrome. UpToDate. Junho, 2007. Disponível em: <http:// www.uptodateonline.com/online/content/search.do>. Acesso em: 28 jul. 2008.

STEAGALL, P.V.M. et al. Evaluation of the adverse effects of oral firocoxib in healthy dogs. Journal of Veterinary Pharmacology and Therapeutics, Oxford, v.30, p.218-223, 2007.

THRALL, M. Hematologia e bioquímica clínica veterinária. São Paulo: Roca, 2007. 582p.

VASCONCELOS, J.T.S. et al. Inibidores seletivos da COX-2 e segurança cardiovascular: onde estamos em 2005? Jornal da LIRNNE, Piauí, v.1, n.2, p.31-37, 2005. 